\title{
Spanning Trees with Few Peripheral Branch Vertices
}

\author{
Pham Hoang Ha*, Dang Dinh Hanh and Nguyen Thanh Loan
}

\begin{abstract}
Let $T$ be a tree, a vertex of degree one is a leaf of $T$ and a vertex of degree at least three is a branch vertex of $T$. The set of leaves of $T$ is denoted by $L(T)$ and the set of branch vertices of $T$ is denoted by $B(T)$. For two distinct vertices $u, v$ of $T$, let $P_{T}[u, v]$ denote the unique path in $T$ connecting $u$ and $v$. Let $T$ be a tree with $B(T) \neq \emptyset$, for each vertex $x \in L(T)$, set $y_{x} \in B(T)$ such that $\left(V\left(P_{T}\left[x, y_{x}\right]\right) \backslash\left\{y_{x}\right\}\right) \cap B(T)=\emptyset$. We delete $V\left(P_{T}\left[x, y_{x}\right]\right) \backslash\left\{y_{x}\right\}$ from $T$ for all $x \in L(T)$. The resulting graph is a subtree of $T$ and is denoted by $\mathrm{R}_{-} \operatorname{Stem}(T)$. It is called the reducible stem of $T$. A leaf of $\mathrm{R}_{-} \operatorname{Stem}(T)$ is called a peripheral branch vertex of $T$. In this paper, we give some sharp sufficient conditions on the independence number and the degree sum for a graph $G$ to have a spanning tree with few peripheral branch vertices.
\end{abstract}

\section{Introduction}

In this paper, we only consider finite simple graphs. Let $G$ be a graph with vertex set $V(G)$ and edge set $E(G)$. For any vertex $v \in V(G)$, we use $N_{G}(v)$ and $\operatorname{deg}_{G}(v)$ (or $N(v)$ and $\operatorname{deg}(v)$ if there is no ambiguity) to denote the set of neighbors of $v$ and the degree of $v$ in $G$, respectively. For any $X \subseteq V(G)$, we denote by $|X|$ the cardinality of $X$. Sometimes, we use $|G|$ (and $G$ ) to denote $|V(G)|$ (and $V(G)$ respectively). We define $N_{G}(X)=\bigcup_{x \in X} N_{G}(x)$ and $\operatorname{deg}_{G}(X)=\sum_{x \in X} \operatorname{deg}_{G}(x)$. We use $G-X$ to denote the graph obtained from $G$ by deleting the vertices in $X$ together with their incident edges. We define $G-u v$ to be the graph obtained from $G$ by deleting the edge $u v \in E(G)$, and $G+u v$ to be the graph obtained from $G$ by adding an edge $u v$ between two non-adjacent vertices $u$ and $v$ of $G$. For two vertices $u$ and $v$ of $G$, the distance between $u$ and $v$ in $G$ is denoted by $d_{G}(u, v)$. We use $K_{n}$ to denote the complete graph on $n$ vertices. We write $A:=B$ to rename $B$ as $A$.

For an integer $m \geq 2$, let $\alpha^{m}(G)$ denote the number defined by

$$
\alpha^{m}(G)=\max \left\{|S|: S \subseteq V(G), d_{G}(x, y) \geq m \text { for all distinct vertices } x, y \in S\right\} .
$$

Received August 14, 2020; Accepted November 29, 2020.

Communicated by Daphne Der-Fen Liu.

2020 Mathematics Subject Classification. 05C05, 05C07, 05 C69.

Key words and phrases. spanning tree, leaf, peripheral branch vertex, independence number, degree sum. *Corresponding author. 
For an integer $p \geq 2$, we define

$$
\begin{array}{r}
\sigma_{p}^{m}(G)=\min \left\{\operatorname{deg}_{G}(S): S \subseteq V(G),|S|=p, d_{G}(x, y) \geq m\right. \\
\\
\text { for all distinct vertices } x, y \in S\} .
\end{array}
$$

For convenience, we define $\sigma_{p}^{m}(G)=+\infty$ if $\alpha^{m}(G)<p$. We note that, $\alpha^{2}(G)$ is often written $\alpha(G)$, which is the independence number of $G$, and $\sigma_{p}^{2}(G)$ is often written $\sigma_{p}(G)$, which is the minimum degree sum of $p$ independent vertices.

Let $T$ be a tree. A vertex of degree one is a leaf of $T$ and a vertex of degree at least three is a branch vertex of $T$. There are several well-known conditions (such as independence number conditions and degree sum conditions) ensuring that a graph $G$ contains a spanning tree with a bounded number of leaves or branch vertices (see 1, 12, 14, 16]). Win [16 obtained a sufficient condition related to the independence number for l-connected graphs, which confirms a conjecture of Las Vergnas [11. Broersma and Tuinstra [1] gave a degree sum condition for a connected graph to contain a spanning tree with at most $k$ leaves.

Theorem 1.1. (see Win [16]) Let $l \geq 1$ and $k \geq 2$ be integers and let $G$ be an l-connected graph. If $\alpha(G) \leq k+l-1$, then $G$ has a spanning tree with at most $k$ leaves.

Theorem 1.2. (see Broerma and Tuinstra [1]) Let $G$ be a connected graph and let $k \geq 2$ be an integer. If $\sigma_{2}(G) \geq|G|-k+1$, then $G$ has a spanning tree with at most $k$ leaves.

The set of leaves of $T$ is denoted by $L(T)$ and the set of branch vertices of $T$ is denoted by $B(T)$. The subtree $T-L(T)$ of $T$ is called the stem of $T$ and is denoted by $\operatorname{Stem}(T)$. Then, many researchers studied spanning trees in connected graphs whose stems have a bounded number of leaves or branch vertices (see $7,8,15,17$ for more details). We introduce here some results on spanning trees whose stems have a few leaves or branch vertices.

Theorem 1.3. (see Tsugaki and Zhang [15]) Let $G$ be a connected graph and let $k \geq 2$ be an integer. If $\sigma_{3}(G) \geq|G|-2 k+1$, then $G$ has a spanning tree whose stem has at most $k$ leaves.

Theorem 1.4. (see Kano and Yan [7]) Let $G$ be a connected graph and let $k \geq 2$ be an integer. If either $\alpha^{4}(G) \leq k$ or $\sigma_{k+1}(G) \geq|G|-k-1$, then $G$ has a spanning tree whose stem has at most $k$ leaves.

Theorem 1.5. (see Kano and Yan [8]) Let $G$ be a connected graph. If $\sigma_{4}^{4}(G) \geq|G|-5$, then $G$ has a spanning tree whose stem is a spider. 
Theorem 1.6. (see Yan [17]) Let $G$ be a connected graph and $k$ be a non-negative integer. If one of the following conditions holds, then $G$ has a spanning tree whose stem has at most $k$ branch vertices.

(a) $\alpha^{4}(G) \leq k+2$,

(b) $\sigma_{k+3}^{4}(G) \geq|G|-2 k-3$.

On the other hand, for a positive integer $t \geq 3$, a graph $G$ is said to be a $K_{1, t}$-free graph if it contains no $K_{1, t}$ as an induced subgraph. If $t=3$, a $K_{1,3}$-free graph is also called a claw-free graph. Many independence number conditions and degree sum conditions ensuring that a $K_{1, t}$-free graph $G$ contains a spanning tree which (or whose stem) has a bounded number of leaves or branch vertices have been derived (see [2,3,5, 6, 9, 10, 13]).

In this paper, we would like to introduce a new concept on spanning tree problem. For two distinct vertices $u$ and $v$ of $T$, let $P_{T}[u, v]$ denote the unique path in $T$ connecting $u$ and $v$. Let $T$ be a tree with $B(T) \neq \emptyset$. For every $x \in L(T)$, set $y_{x} \in B(T)$ such that $\left(V\left(P_{T}\left[x, y_{x}\right]\right) \backslash\left\{y_{x}\right\}\right) \cap B(T)=\emptyset$. We delete $V\left(P_{T}\left[x, y_{x}\right]\right) \backslash\left\{y_{x}\right\}$ from $T$ for all $x \in L(T)$. The resulting graph is denoted by $\mathrm{R}_{-} \mathrm{Stem}(T)$. It is called the reducible stem of $T$. The path that connects $x$ to $y_{x}$ but does not contain $y_{x}$, is called a leaf-branch path of $T$ incident to $x$ and denoted by $B_{x}$. Let $B=\bigcup_{x \in L(T)} V\left(B_{x}\right)$, then R_Stem $(T)=T-B$ (see Figure 1.1 for an example of $T$ and R_Stem $(T))$.
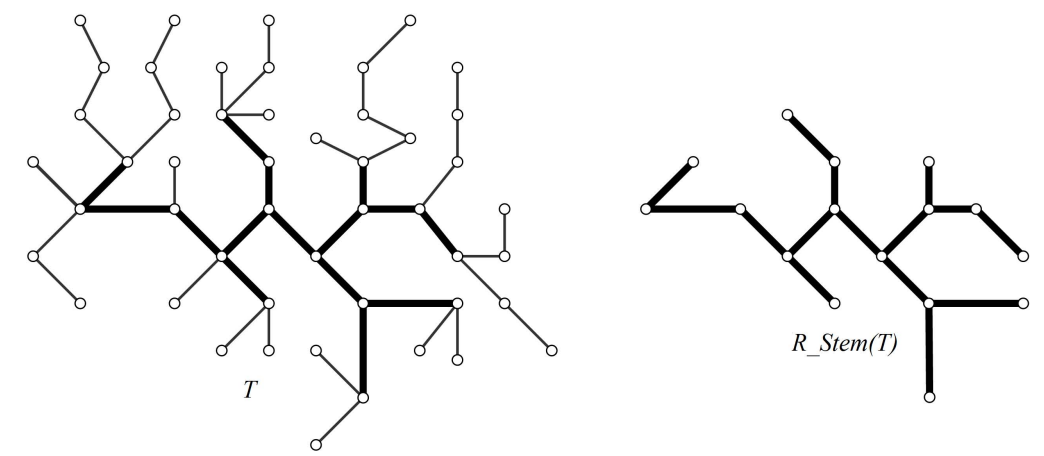

Figure 1.1: Tree $T$ and R_Stem $(T)$.

A leaf of R_Stem $(T)$ is also called a peripheral branch vertex of $T$ (see $[12)$. We denote by $P(B(T))$ the peripheral branch vertex set of $T$. Then $P(B(T))=L($ R_Stem $(T))$.

We would like to study sufficient conditions for a graph to have a spanning tree $T$ with few peripheral branch vertices, i.e., R_Stem $(T)$ has a few leaves. In particular, we state the following theorem. 
Theorem 1.7. Let $G$ be a connected graph and let $k \geq 2$ be an integer. If one of the following conditions holds, then $G$ has a spanning tree with at most $k$ peripheral branch vertices.

(i) $\alpha(G) \leq 2 k+2$,

(ii) $\sigma_{k+1}^{4}(G) \geq\left\lfloor\frac{|G|-k}{2}\right\rfloor$.

Here, the notation $\lfloor r\rfloor$ stands for the biggest integer that does not exceed the real number $r$.

To end this section, we give an example to show that our main results are sharp. Let $k \geq 2$ and $m \geq 1$ be integers, and let $D_{1}, D_{2}, \ldots, D_{k+1}$ and $H_{1}, H_{2}, \ldots, H_{k+1}$ be $2 k+2$ disjoint copies of the complete graph $K_{m}$ of order $m$. Let $w, x_{1}, x_{2}, \ldots, x_{k+1}$ be $k+2$ vertices not contained in $V\left(D_{1}\right) \cup V\left(D_{2}\right) \cup \cdots \cup V\left(D_{k+1}\right) \cup V\left(H_{1}\right) \cup V\left(H_{2}\right) \cup \cdots \cup V\left(H_{k+1}\right)$. Join $w$ to all vertices of $\left\{x_{1}, x_{2}, \ldots, x_{k+1}\right\}$ and join $x_{i}$ to all the vertices in $V\left(D_{i}\right) \cup V\left(H_{i}\right)$ for every $1 \leq i \leq k+1$. Let $G$ denote the resulting graph (see Figure 1.2 ). Then $\alpha(G)=2 k+3$.

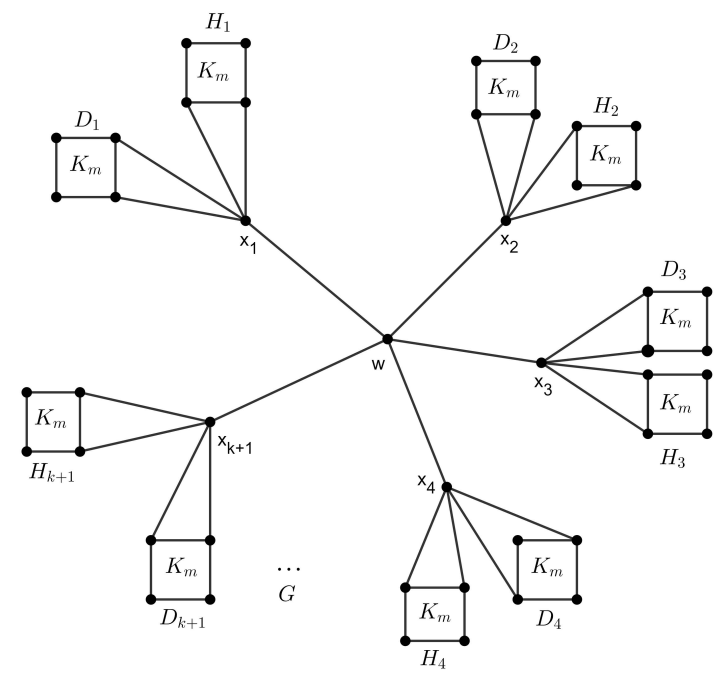

Figure 1.2: Graph $G$.

Moreover, let $S$ be a subset of $V(G)$ such that $|S|=k+1$ and $d_{G}(x, y) \geq 4$ for all distinct vertices $x, y \in S$, then $S \cap\left(V\left(D_{i}\right) \cup V\left(H_{i}\right)\right) \neq \emptyset$ for every $1 \leq i \leq k+1$. Therefore, for every $1 \leq i \leq k+1$, take $y_{i} \in V\left(D_{i}\right) \cup V\left(H_{i}\right)$. We then obtain

$$
\sigma_{k+1}^{4}(G)=\sum_{i=1}^{k+1} \operatorname{deg}_{G}\left(y_{i}\right)=(k+1) m=\left\lfloor\frac{|G|-k}{2}\right\rfloor-1 .
$$

But $G$ has no spanning tree with at most $k$ peripheral branch vertices. Then, our main results are sharp. 
Since $\sigma_{k+1}(G) \leq \sigma_{k+1}^{4}(G)$, we have a corollary of Theorem 1.7 as follows.

Corollary 1.8. Let $G$ be a connected graph and let $k \geq 2$ be an integer. If $\sigma_{k+1}(G) \geq$ $\left\lfloor\frac{|G|-k}{2}\right\rfloor$, then $G$ has a spanning tree with at most $k$ peripheral branch vertices.

We also note that in the above example, if $m \leq k+1$ then $\sigma_{k+1}(G)=\sigma_{k+1}^{4}(G)=$ $\left\lfloor\frac{|G|-k}{2}\right\rfloor-1$. So, the condition $\sigma_{k+1}(G) \geq\left\lfloor\frac{|G|-k}{2}\right\rfloor$ of Corollary 1.8 is tight.

\section{Proof of the main result}

Let $T$ be a tree. For two distinct vertices $u$ and $v$ of $T$, we always define the orientation of $P_{T}[u, v]$ to be from $u$ to $v$. If $v \in V(P)$, then $v^{+}$and $v^{-}$denote the successor and predecessor of $v$ on $P$ if they exist, respectively. For any $X \subseteq V(G)$, set $(N(X) \cap$ $\left.P_{T}[u, v]\right)^{-}=\left\{x^{-} \mid x \in V\left(P_{T}[u, v]\right) \backslash\{u\}\right.$ and $\left.x \in N(X)\right\}$ and $\left(N(X) \cap P_{T}[u, v]\right)^{+}=\left\{x^{+} \mid\right.$ $x \in V\left(P_{T}[u, v]\right) \backslash\{v\}$ and $\left.x \in N(X)\right\}$. For an integer $t \geq 1$, we let $N_{t}(X)=\{x \in V(G) \mid$ $|N(x) \cap X|=t\}$. We refer to [4] for terminology and notation not defined here.

Proof of Theorem 1.7. Suppose, to the contrary, each spanning tree of $G$ contains at least $k+1$ peripheral branch vertices. Let $\mathcal{T}=\{T: T$ is a subgraph of $G$ and $T$ is a tree $\}$, and let $\mathcal{T}_{k+1}=\{T: T \in \mathcal{T}$ and $|P(B(T))|=k+1\}$. Choose a maximal tree $T$ in $\mathcal{T}_{k+1}$ (a tree $T$ in $\mathcal{T}_{k+1}$ such that $|V(T)|$ is maximum) which satisfies the following two conditions:

(C1) $\mid$ R_Stem $(T) \mid$ is as small as possible,

(C2) $|L(T)|$ is as small as possible subject to (C1).

Claim 2.1. There does not exist a tree $S$ in $G$ such that $V(S)=V(T)$ and $|P(B(S))| \leq k$.

Proof. Suppose, to the contrary, there exists a tree $S$ in $G$ such that $V(S)=V(T)$ and $|P(B(S))| \leq k$. Since $|P(B(S))| \leq k, S$ is not a spanning tree of $G$. Then there exists $u \in V(G)-V(S)$ such that $u$ is adjacent to a vertex $v \in S$. Let $S_{1}$ be a tree obtained from $S$ by adding the edge $u v$. Then $S_{1}$ is a tree in $G$ such that $\left|V\left(S_{1}\right)\right|=|V(T)|+1$ and $\left|P\left(B\left(S_{1}\right)\right)\right| \leq k+1$.

If $\left|P\left(B\left(S_{1}\right)\right)\right|=k+1$, then $S_{1}$ contradicts the maximality of $T$ (since $\left|V\left(S_{1}\right)\right|=$ $|V(S)|+1=|V(T)|+1>|V(T)|)$. So we may assume that $\left|P\left(B\left(S_{1}\right)\right)\right| \leq k$. By repeating this process, we can recursively construct a set of trees $\left\{S_{i} \mid i \geq 1\right\}$ in $G$ such that $S_{i}$ satisfies that $\left|P\left(B\left(S_{i}\right)\right)\right| \leq k$ and $\left|V\left(S_{i+1}\right)\right|=\left|V\left(S_{i}\right)\right|+1$ for each $i \geq 1$. Since $G$ has no spanning tree with at most $k$ peripheral branch vertices and $|V(G)|$ is finite, the process must terminate after a finite number of steps, i.e., there exists some $h \geq 1$ such that $S_{h+1}$ is a tree in $G$ with $\left|P\left(B\left(S_{h+1}\right)\right)\right|=k+1$. But this contradicts the maximality of $T$. So the claim holds. 
Set $P(B(T))=\left\{x_{1}, x_{2}, \ldots, x_{k+1}\right\}$. By the definition of peripheral branch vertex, we have the following claim.

Claim 2.2. For every $i \in\{1,2, \ldots, k+1\}$, there exist at least two leaf-branch paths of $T$ which are incident to $x_{i}$.

Now we will prove the following two claims to show that $\alpha(G) \geq 2 k+3$.

Claim 2.3. For each $i \in\{1,2, \ldots, k+1\}$, there exist $y_{i}, z_{i} \in L(T)$ such that $B_{y_{i}}, B_{z_{i}}$ are incident to $x_{i}$ and $N_{G}\left(y_{i}\right) \cap\left(V\left(\mathrm{R} \_\operatorname{Stem}(T)\right)-\left\{x_{i}\right\}\right)=\emptyset$ and $N_{G}\left(z_{i}\right) \cap\left(V\left(\mathrm{R} \_\operatorname{Stem}(T)\right)-\right.$ $\left.\left\{x_{i}\right\}\right)=\emptyset$.

Proof. Let $\left\{a_{i j}\right\}_{j=1}^{m}$ be the subset of $L(T)$ such that $B_{a_{i j}}$ is incident to $x_{i}$. By Claim 2.2. we obtain $m \geq 2$.

Suppose that there are more than $m-2$ vertices in $\left\{a_{i j}\right\}_{j=1}^{m}$ satisfying

$$
N_{G}\left(a_{i j}\right) \cap\left(V(\text { R_Stem }(T))-\left\{x_{i}\right\}\right) \neq \emptyset .
$$

Without loss of generality, we may assume that $N_{G}\left(a_{i j}\right) \cap\left(V(\right.$ R_Stem $\left.(T))-\left\{x_{i}\right\}\right) \neq \emptyset$ for all $j=2, \ldots, m$. Set $b_{i j} \in N_{G}\left(a_{i j}\right) \cap\left(V(\right.$R_Stem $\left.(T))-\left\{x_{i}\right\}\right)$ and $v_{i j} \in N_{T}\left(x_{i}\right) \cap V\left(P_{T}\left[a_{i j}, x_{i}\right]\right)$ for all $j \in\{2, \ldots, m\}$. Consider the tree

$$
T^{\prime}:=T+\left\{a_{i j} b_{i j}\right\}_{j=2}^{m}-\left\{x_{i} v_{i j}\right\}_{j=2}^{m}
$$

Then $T^{\prime}$ satisfies $\left|V\left(T^{\prime}\right)\right|=|V(T)|,\left|P\left(B\left(T^{\prime}\right)\right)\right| \leq|P(B(T))|$ and $\mid$ R_Stem $_{-}\left(T^{\prime}\right) \mid<$ $\mid$ R_Stem $(T) \mid$, where $x_{i}$ is not in $V\left(R_{-} \_\right.$Stem $\left.\left(T^{\prime}\right)\right)$. This contradicts either Claim 2.1 or Condition (C1). Therefore, Claim 2.3 holds.

Set $U=\left\{y_{i}, z_{i}\right\}_{i=1}^{k+1}$. By the maximality of $T$ we have $N_{G}(U) \subseteq V(T)$.

Claim 2.4. $U$ is an independent set in $G$.

Proof. Suppose that there exist two vertices $u, v \in U$ such that $u v \in E(G)$. Without loss of generality, we may assume that $v=y_{i}$ for some $i \in\{1,2, \ldots, k+1\}$. Set $v_{i} \in N_{T}\left(x_{i}\right) \cap$ $V\left(B_{y_{i}}\right)$. Consider the tree $T^{\prime}:=T+u y_{i}-v_{i} x_{i}$. Then $V\left(T^{\prime}\right)=V(T)$ and $\left|P\left(B\left(T^{\prime}\right)\right)\right| \leq$ $|P(B(T))|$. If $\operatorname{deg}_{T}\left(x_{i}\right)=3$ then $x_{i}$ is not a branch vertex of $T^{\prime}$. Hence $\mid$ R_Stem $\left(T^{\prime}\right) \mid<$ $\mid$ R_Stem $(T) \mid$, this contradicts either Claim 2.1 or Condition (C1). Otherwise, we have $\left|P\left(B\left(T^{\prime}\right)\right)\right|=|P(B(T))|, \mid$ R_Stem $\left(T^{\prime}\right)|=|$ R_Stem $(T) \mid$ and $\left|L\left(T^{\prime}\right)\right|<|L(T)|$, where either $T^{\prime}$ has only one new leaf and $y_{i}, u$ are not leaves of $T^{\prime}$ or $y_{i}$ is still a leaf of $T^{\prime}$ but $T^{\prime}$ has no new leaf and $u$ is not a leaf of $T^{\prime}$. This contradicts Condition (C2). The proof of Claim 2.4 is completed.

Since $k \geq 2$, then $\left|L\left(\mathrm{R} \_S t e m(T)\right)\right|=|P(B(T))| \geq 3$. Hence, we have $\left|B\left(\mathrm{R}_{-} \mathrm{Stem}(T)\right)\right|$ $\geq 1$. Let $u$ be a vertex in $B\left(R_{-}\right.$Stem $\left.(T)\right)$. By Claims 2.3 and 2.4, we conclude that $U \cup\{u\}$ 
is an independent set in $G$. This implies that $\alpha(G) \geq 2 k+3$. As either $\alpha(G) \leq 2 k+2$, or $\sigma_{k+1}^{4}(G) \geq\left\lfloor\frac{|G|-k}{2}\right\rfloor$, we conclude that $\sigma_{k+1}^{4}(G) \geq\left\lfloor\frac{|G|-k}{2}\right\rfloor$.

Claim 2.5. For every $i, j \in\{1,2, \ldots, k+1\}$ where $i \neq j, N_{G}\left(y_{i}\right) \cap V\left(B_{y_{j}}\right)=\emptyset$ and $N_{G}\left(y_{i}\right) \cap V\left(B_{z_{j}}\right)=\emptyset$.

Proof. By the same role of $y_{j}$ and $z_{j}$, we only need to prove $N_{G}\left(y_{i}\right) \cap V\left(B_{y_{j}}\right)=\emptyset$. Suppose the assertion of the claim is false. Then there exists a vertex $x \in N_{G}\left(y_{i}\right) \cap V\left(B_{y_{j}}\right)$. Set $T^{\prime}:=T+x y_{i}$. Then $T^{\prime}$ is a subgraph of $G$ including a unique cycle $C$, which contains both $x_{i}$ and $x_{j}$.

Since $k \geq 2$, then $\mid L\left(\mathrm{R} \_\right.$Stem $\left.(T)\right)|=| P(B(T)) \mid \geq 3$. Hence, we obtain $\mid B\left(\mathrm{R} \_\right.$Stem $\left.(T)\right) \mid$ $\geq 1$. Then there exists a branch vertex of R_Stem $(T)$ contained in $C$. Let $e$ be an edge incident to such a vertex in $C$ and R_Stem $(T)$. By removing the edge $e$ from $T^{\prime}$ we obtain a tree $T^{\prime \prime}$ of $G$ satisfying $V\left(T^{\prime \prime}\right)=V(T)$ and $\left|P\left(B\left(T^{\prime \prime}\right)\right)\right| \leq k$, the reason is that either R_Stem $\left(T^{\prime \prime}\right)$ has only one new leaf and $x_{i}, x_{j}$ are not leaves of R_Stem( $\left(T^{\prime \prime}\right)$ or $x_{i}$ (or $\left.x_{j}\right)$ is still a leaf of R_Stem $\left(T^{\prime \prime}\right)$ but R_Stem $\left(T^{\prime \prime}\right)$ has no new leaf and $x_{j}$ (or $x_{i}$ respectively) is not a leaf of R_Stem $\left(T^{\prime \prime}\right)$. This is a contradiction with Claim 2.1. So Claim 2.5 is proved.

Claim 2.6. For every $1 \leq i<j \leq k+1, d_{G}\left(y_{i}, y_{j}\right) \geq 4$ and $d_{G}\left(z_{i}, z_{j}\right) \geq 4$.

Proof. We first prove that $d_{G}\left(y_{i}, y_{j}\right) \geq 4$. Let $P\left[y_{i}, y_{j}\right]$ be a shortest path connecting $y_{i}$ and $y_{j}$ in $G$. Assume that all vertices of $P\left[y_{i}, y_{j}\right]$ are contained in $\left(V(G)-V\left(\mathrm{R}_{-} \operatorname{Stem}(T)\right)\right) \cup$ $\left\{x_{i}, x_{j}\right\}$.

Let $t_{i} \in B_{y_{i}} \cup\left\{x_{i}\right\}, t_{j} \in B_{y_{j}} \cup\left\{x_{j}\right\}$ such that $t_{i}, t_{j} \in P\left[y_{i}, y_{j}\right]$ and

$$
P_{P\left[y_{i}, y_{j}\right]}\left[t_{i}, t_{j}\right] \cap B_{y_{i}}=\left\{t_{i}\right\}, \quad P_{P\left[y_{i}, y_{j}\right]}\left[t_{i}, t_{j}\right] \cap B_{y_{j}}=\left\{t_{j}\right\}
$$

Set $P\left[t_{i}, t_{j}\right]:=P_{P\left[y_{i}, y_{j}\right]}\left[t_{i}, t_{j}\right]$. For every vertex $p \in L(T)$ such that $B_{p} \cap P\left[t_{i}, t_{j}\right] \neq \emptyset$. Let $v_{p} \in B(T)$ such that $\left(V\left(P_{T}\left[p, v_{p}\right]\right) \backslash\left\{v_{p}\right\}\right) \cap B(T)=\emptyset$. Let $v_{p}^{-} \in V\left(B_{p}\right) \cap N_{T}\left(v_{p}\right)$. Remove all the edges $v_{p} v_{p}^{-}$of $T$ and add $P\left[t_{i}, t_{j}\right]$. Then the resulting subgraph $T^{\prime}$ of $G$ includes a unique cycle $C$, which contains the vertices $x_{i}$ and $x_{j}$. Since $k \geq 2$, then $\left|L\left(\mathrm{R} \_\operatorname{Stem}(T)\right)\right|=|P(B(T))| \geq 3$. Hence, we obtain $\left|B\left(\mathrm{R} \_\operatorname{Stem}(T)\right)\right| \geq 1$. Then, there exists a branch vertex $u$ of R_Stem $(T)$ contained in $C$. Let $e$ be an edge in $C$ which is incident to $u$. Denote by $T^{\prime \prime}$ the tree obtained from $T^{\prime}$ by removing the edge $e$ (see Figure 2.1). Then $V(T) \subseteq V\left(T^{\prime}\right)=V\left(T^{\prime \prime}\right)$ and $\left|P\left(B\left(T^{\prime \prime}\right)\right)\right| \leq k$, where either R_Stem( $\left(T^{\prime \prime}\right)$ has only one new leaf and $x_{i}, x_{j}$ are not leaves of $\mathrm{R} \_\operatorname{Stem}\left(T^{\prime \prime}\right)$ or $x_{i}$ (or $x_{j}$ ) is still a leaf of R_Stem $\left(T^{\prime \prime}\right)$ but R_Stem $\left(T^{\prime \prime}\right)$ has no new leaf and $x_{j}$ (or $x_{i}$ respectively) is not a leaf of $\mathrm{R} \_$Stem $\left(T^{\prime \prime}\right)$. This contradicts either the maximality of $T$ or Claim 2.1. Therefore, 
$P\left[y_{i}, y_{j}\right] \cap\left(\mathrm{R} \_\operatorname{Stem}(T)-\left\{x_{i}, x_{j}\right\}\right) \neq \emptyset$. Set $v \in P\left[y_{i}, y_{j}\right] \cap\left(\mathrm{R} \_\operatorname{Stem}(T)-\left\{x_{i}, x_{j}\right\}\right)$. Hence, by combining with Claim 2.3 , we obtain

$$
d_{G}\left(y_{i}, y_{j}\right)=d_{P\left[y_{i}, y_{j}\right]}\left(y_{i}, y_{j}\right) \geq d_{P\left[y_{i}, y_{j}\right]}\left(y_{i}, v\right)+d_{P\left[y_{i}, y_{j}\right]}\left(v, y_{j}\right) \geq 2+2=4
$$

Now, using the same arguments, we also obtain that $d_{G}\left(z_{i}, z_{j}\right) \geq 4$. This completes the

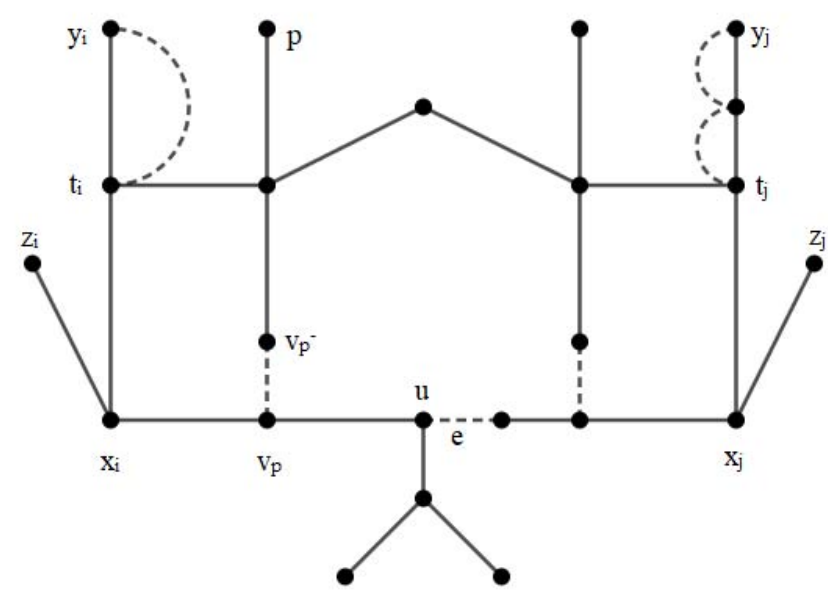

Figure 2.1: Tree $T^{\prime \prime}$.

proof of Claim 2.6.

Claim 2.7. If $p \in L(T)-U$, then $\sum_{u \in U}\left|N_{G}(u) \cap B_{p}\right| \leq\left|B_{p}\right|-1$.

Proof. Set $v_{p} \in B(T)$ such that $\left(V\left(P_{T}\left[p, v_{p}\right]\right) \backslash\left\{v_{p}\right\}\right) \cap B(T)=\emptyset$. Let $V\left(B_{p}\right) \cap N_{T}\left(v_{p}\right)=$ $\left\{v_{p}^{-}\right\}$. Then we consider $B_{p}=P_{T}\left[p, v_{p}^{-}\right]$.

Subclaim 2.7.1. For every $i \in\{1,2, \ldots, k+1\}$, if $x \in N_{G}\left(y_{i}\right) \cap B_{p}$ then $x^{-} \notin N_{G}(U-$ $\left.\left\{y_{i}\right\}\right) \cap B_{p}$.

Suppose that there exists $x^{-} \in N_{G}(z) \cap B_{p}$ with $z \in U-\left\{y_{i}\right\}$. Let $T^{\prime}:=T+\left\{x y_{i}, x^{-} z\right\}-$ $\left\{x x^{-}, v_{p} v_{p}^{-}\right\}$. Then $T^{\prime}$ is a tree in $G$ satisfying $V\left(T^{\prime}\right)=V(T),\left|P\left(B\left(T^{\prime}\right)\right)\right|=|P(B(T))|$, $\mid$ R_Stem $\left(T^{\prime}\right)|=|$ R_Stem $(T) \mid$ and $\left|L\left(T^{\prime}\right)\right|<|L(T)|$, where $y_{i}$, $z$ are not leaves of $T^{\prime}$ (see Figure 2.2. Hence this contradicts Condition (C2).

Subclaim 2.7.2. If $x \in B_{p}$, then $x$ is adjacent to at most 2 vertices in $U$.

Indeed, we can prove a stronger statement that if $x \in N_{G}\left(y_{i}\right) \cap B_{p}$ then $x \notin N_{G}\left(y_{j}\right) \cap B_{p}$ and $x \notin N_{G}\left(z_{j}\right) \cap B_{p}$ for all $1 \leq i, j \leq k+1, i \neq j$. Suppose, to the contrary, there exist $i$ and $j$, with $1 \leq i, j \leq k+1, i \neq j$, such that $x \in N_{G}\left(y_{i}\right) \cap B_{p}$ and $x \in N_{G}(w)$, where $w=y_{j}$ or $w=z_{j}$. Without loss of generality, we assume that $w=y_{j}$. Set $T^{\prime}:=T+\left\{x y_{i}, x y_{j}\right\}-\left\{v_{p} v_{p}^{-}\right\}$. Then $T^{\prime}$ is a subgraph of $G$ that includes a unique cycle $C$, which contains two vertices $x_{i}$ and $x_{j}$. Since $k \geq 2$, then $\left|L\left(\mathrm{R} \_\operatorname{Stem}(T)\right)\right|=|P(B(T))| \geq 3$. 


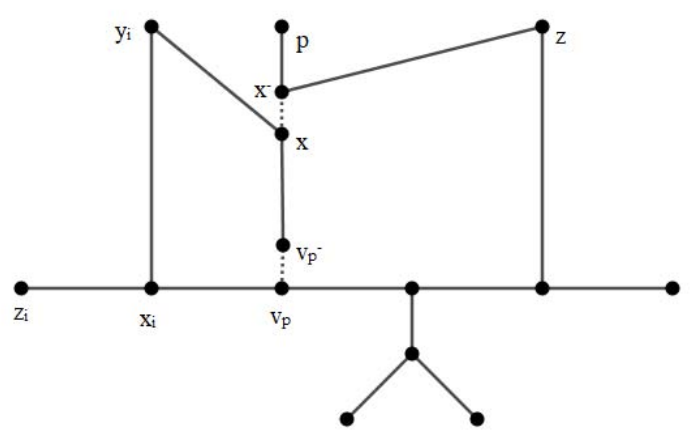

Figure 2.2: Tree $T^{\prime}$.

Hence, we obtain $\mid B\left(\right.$ R_Stem $\left._{-}(T)\right) \mid \geq 1$. Then, there exists a branch vertex of R_Stem(T) contained in $C$. Let $e$ be an edge which is incident to such a vertex in $C$. By removing the edge $e$ we obtain a tree $T^{\prime \prime}$ of $G$ (see Figure 2.3).

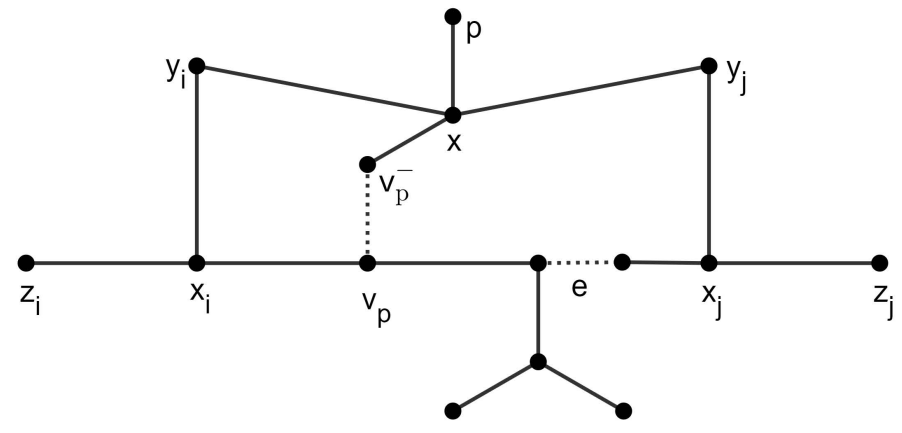

Figure 2.3: Tree $T^{\prime \prime}$.

Then $V\left(T^{\prime \prime}\right)=V(T)$ and $\left|P\left(B\left(T^{\prime \prime}\right)\right)\right| \leq k$, where $x_{i}$ and $x_{j}$ are not leaves of R_Stem $\left(T^{\prime \prime}\right)$. This contradicts either the maximality of $T$ or Claim 2.1. Therefore, we have $\left|U \cap N_{G}(x)\right| \leq$ 2. The proof of Subclaim 2.7.2 is completed.

Subclaim 2.7.3. $p \notin N_{G}(U)$ and $v_{p}^{-} \notin N_{G}(U)$.

Suppose, to the contrary, $z \in N_{G}\left(y_{i}\right)$ for some $z \in\left\{p, v_{p}^{-}\right\}$and $y_{i} \in U$. Consider the tree $T^{\prime}:=T+y_{i} z-v_{p} v_{p}^{-}$. Then $T^{\prime}$ is a tree in $G$ satisfying $V\left(T^{\prime}\right)=V(T)$, $\left|P\left(B\left(T^{\prime}\right)\right)\right|=|P(B(T))|, \mid R_{-}$Stem $\left(T^{\prime}\right)|=| R_{-} \_\operatorname{Stem}(T) \mid$ and $\left|L\left(T^{\prime}\right)\right|<|L(T)|$. This contradicts Condition (C2). Therefore, Subclaim 2.7.3 holds.

Now, by Subclaims 2.7.1-2.7.3 we conclude that $\{p\}, N_{G}\left(y_{i}\right) \cap B_{p},\left(N_{G}\left(U-\left\{y_{i}\right\}\right) \cap B_{p}\right)^{+}$ and $\left(N_{2}(U)-N\left(y_{i}\right)\right) \cap B_{p}$ are pairwise disjoint subsets in $B_{p}$ for every $1 \leq i \leq k+1$. Recall that $N_{3}(U) \cap B_{p}=\emptyset$ by Subclaim 2.7.2. Then by combining with Subclaim 2.7.3 
we obtain

$$
\begin{aligned}
\sum_{u \in U}\left|N_{G}(u) \cap B_{p}\right| & =\left|N_{G}\left(y_{i}\right) \cap B_{p}\right|+\left|N_{G}\left(U-\left\{y_{i}\right\}\right) \cap B_{p}\right|+\left|\left(N_{2}(U)-N\left(y_{i}\right)\right) \cap B_{p}\right| \\
& =\left|N_{G}\left(y_{i}\right) \cap B_{p}\right|+\left|\left(N_{G}\left(U-\left\{y_{i}\right\}\right) \cap B_{p}\right)^{+}\right|+\left|\left(N_{2}(U)-N\left(y_{i}\right)\right) \cap B_{p}\right| \\
& \leq\left|B_{p}\right|-1 .
\end{aligned}
$$

Claim 2.7 is proved.

Claim 2.8. For every $1 \leq i \leq k+1, \sum_{u \in U}\left|N_{G}(u) \cap B_{y_{i}}\right| \leq\left|B_{y_{i}}\right|-1$ and $\sum_{u \in U} \mid N_{G}(u) \cap$ $B_{z_{i}}|\leq| B_{z_{i}} \mid-1$.

Proof. By the same role of $y_{i}$ and $z_{i}$, we only need to prove $\sum_{u \in U}\left|N_{G}(u) \cap B_{y_{i}}\right| \leq\left|B_{y_{i}}\right|-1$. Set $V\left(B_{y_{i}}\right) \cap N_{T}\left(x_{i}\right)=\left\{x_{i}^{-}\right\}$. Now we consider $B_{y_{i}}=P_{T}\left[y_{i}, x_{i}^{-}\right]$.

By Claim 2.5, we obtain the following.

Subclaim 2.8.1. $N_{G}(U) \cap B_{y_{i}}=N_{G}\left(\left\{y_{i}, z_{i}\right\}\right) \cap B_{y_{i}}$.

Subclaim 2.8.2. If $x \in N_{G}\left(y_{i}\right) \cap B_{y_{i}}$ then $x^{-} \notin N_{G}\left(z_{i}\right) \cap B_{y_{i}}$.

Suppose that there exists $x \in N_{G}\left(y_{i}\right) \cap B_{y_{i}}$ such that $x^{-} \in N_{G}\left(z_{i}\right) \cap B_{y_{i}}$. Consider the tree $T^{\prime}:=T+\left\{x y_{i}, z_{i} x^{-}\right\}-\left\{x x^{-}, x_{i}^{-} x_{i}\right\}$. Then $V\left(T^{\prime}\right)=V(T)$ and $\left|P\left(B\left(T^{\prime}\right)\right)\right| \leq$ $|P(B(T))|$. If $\operatorname{deg}_{T}\left(x_{i}\right)=3$ then $x_{i}$ is not a branch vertex of $T^{\prime}$. Hence $\left|\mathrm{R}_{-} \operatorname{Stem}\left(T^{\prime}\right)\right|<$ $\mid$ R_Stem $(T) \mid$, this contradicts either Claim 2.1 or Condition (C1). Otherwise, we have $\left|P\left(B\left(T^{\prime}\right)\right)\right|=|P(B(T))|,\left|\mathrm{R} \_S t e m\left(T^{\prime}\right)\right|=\left|\mathrm{R}_{-} \operatorname{Stem}(T)\right|$ and $\left|L\left(T^{\prime}\right)\right|<|L(T)|$, where $y_{i}$ and $z_{i}$ are not leaves of $T^{\prime}$. This is a contradiction with Condition (C2). Therefore, Subclaim 2.8.2 holds.

Subclaim 2.8.3. $x_{i}^{-} \notin N_{G}\left(z_{i}\right)$.

Suppose, to the contrary, $x_{i}^{-} z_{i} \in E(G)$. Consider the tree $T^{\prime}:=T+x_{i}^{-} z_{i}-x_{i} x_{i}^{-}$. Then $T^{\prime}$ is a tree in $G$ satisfying $V\left(T^{\prime}\right)=V(T),\left|P\left(B\left(T^{\prime}\right)\right)\right|=|P(B(T))|, \mid$ R_Stem $\left(T^{\prime}\right) \mid=$ $\mid$ R_Stem $(T) \mid$ and $\left|L\left(T^{\prime}\right)\right|<|L(T)|$, where $z_{i}$ is not a leaf of $T^{\prime}$. This contradicts Condition (C2). Therefore, Subclaim 2.8.3 holds.

By Subclaims 2.8.1-2.8.3, we conclude that $\left\{y_{i}\right\}, N_{G}\left(y_{i}\right) \cap B_{y_{i}}$ and $\left(N_{G}\left(z_{i}\right) \cap B_{y_{i}}\right)^{+}$ are pairwise disjoint subsets in $B_{y_{i}}$. Combining with Subclaim 2.8.1, we have

$$
\begin{aligned}
\sum_{u \in U}\left|N_{G}(u) \cap B_{y_{i}}\right| & =\left|N_{G}\left(y_{i}\right) \cap B_{y_{i}}\right|+\left|N_{G}\left(z_{i}\right) \cap B_{y_{i}}\right| \\
& =\left|N_{G}\left(y_{i}\right) \cap B_{y_{i}}\right|+\left|\left(N_{G}\left(z_{i}\right) \cap B_{y_{i}}\right)^{+}\right| \leq\left|B_{y_{i}}\right|-1 .
\end{aligned}
$$

This completes the proof of Claim 2.8.

By Claims 2.3, 2.7 and 2.8, we obtain that

$$
\operatorname{deg}_{G}(U)=\sum_{i=1}^{k+1}\left(\operatorname{deg}_{G}\left(y_{i}\right)+\operatorname{deg}_{G}\left(z_{i}\right)\right)
$$




$$
\begin{aligned}
& \leq \sum_{i=1}^{k+1}\left(\left|B_{y_{i}}\right|-1\right)+\sum_{i=1}^{k+1}\left(\left|B_{z_{i}}\right|-1\right)+\sum_{p \in L(T)-U}\left(\left|B_{p}\right|-1\right)+2(k+1) \\
& =|G|-\left|\mathrm{R} \_\operatorname{Stem}(T)\right|-|L(T)-U| \\
& \leq|G|-\left|\mathrm{R} \_\operatorname{Stem}(T)\right| .
\end{aligned}
$$

On the other hand, since $k \geq 2$, then $\mid L\left(\right.$ R_Stem $\left._{(T)}\right)|=| P(B(T)) \mid=k+1 \geq 3$. Hence, we obtain $\mid B($ R_Stem $(T)) \mid \geq 1$. So we have $\mid$ R_Stem $(T) \mid \geq k+2$. Hence

$$
\begin{aligned}
& \sum_{i=1}^{k+1} \operatorname{deg}_{G}\left(y_{i}\right)+\sum_{i=1}^{k+1} \operatorname{deg}_{G}\left(z_{i}\right) \leq|G|-k-2 \\
\Longrightarrow & \min \left\{\sum_{i=1}^{k+1} \operatorname{deg}_{G}\left(y_{i}\right), \sum_{i=1}^{k+1} \operatorname{deg}_{G}\left(z_{i}\right)\right\} \leq\left\lfloor\frac{|G|-k-2}{2}\right\rfloor .
\end{aligned}
$$

Combining with Claim 2.6, we obtain

$$
\sigma_{k+1}^{4}(G) \leq \min \left\{\sum_{i=1}^{k+1} \operatorname{deg}_{G}\left(y_{i}\right), \sum_{i=1}^{k+1} \operatorname{deg}_{G}\left(z_{i}\right)\right\} \leq\left\lfloor\frac{|G|-k}{2}\right\rfloor-1 .
$$

Thus, $G$ does not satisfy either the condition $\alpha(G) \leq 2 k+2$, or the condition $\sigma_{k+1}^{4}(G) \geq$ $\left\lfloor\frac{|G|-k}{2}\right\rfloor$, a contradiction. Therefore, $G$ has a spanning tree with at most $k$ peripheral branch vertices if either $\alpha(G) \leq 2 k+2$, or $\sigma_{k+1}^{4}(G) \geq\left\lfloor\frac{|G|-k}{2}\right\rfloor$.

The proof of Theorem 1.7 is completed.

\section{Acknowledgments}

A part of work was completed during a stay of the first named author at the Vietnam Institute for Advanced Study in Mathematics (VIASM). He would like to thank this institution for financial support and hospitality. This research is funded by Vietnam National Foundation for Science and Technology Development (NAFOSTED) under grant number 101.04-2018.03.

\section{References}

[1] H. Broersma and H. Tuinstra, Independence trees and Hamilton cycles, J. Graph Theory 29 (1998), no. 4, 227-237.

[2] Y. Chen, G. Chen and Z. Hu, Spanning 3 -ended trees in $k$-connected $K_{1,4}$-free graphs, Sci. China Math. 57 (2014), no. 8, 1579-1586.

[3] Y. Chen, P. H. Ha and D. D. Hanh, Spanning trees with at most 4 leaves in $K_{1,5}$-free graphs, Discrete Math. 342 (2019), no. 8, 2342-2349. 
[4] R. Diestel, Graph Theory, Third edition, Graduate Texts in Mathematics 173, Springer-Verlag, Berlin, 2005.

[5] P. H. Ha and D. D. Hanh, Spanning trees of connected $K_{1, t}$-free graphs whose stems have a few leaves, Bull. Malays. Math. Sci. Soc. 43 (2020), no. 3, 2373-2383.

[6] M. Kano, A. Kyaw, H. Matsuda, K. Ozeki, A. Saito and T. Yamashita, Spanning trees with a bounded number of leaves in a claw-free graph, Ars Combin. 103 (2012), 137-154.

[7] M. Kano and Z. Yan, Spanning trees whose stems have at most $k$ leaves, Ars Combin. 117 (2014), 417-424.

[8] _ Spanning trees whose stems are spiders, Graphs Combin. 31 (2015), no. 6, 1883-1887.

[9] A. Kyaw, Spanning trees with at most 3 leaves in $K_{1,4}$-free graphs, Discrete Math. 309 (2009), no. 20, 6146-6148.

[10] _ Spanning trees with at most $k$ leaves in $K_{1,4}$-free graphs, Discrete Math. 311 (2011), no. 20, 2135-2142.

[11] M. Las Vergnas, Sur une propriété des arbres maximaux dans un graphe, C. R. Acad. Sci. Paris Sér. A-B 272 (1971), A1297-A1300.

[12] S.-i. Maezawa, R. Matsubara and H. Matsuda, Degree conditions for graphs to have spanning trees with few branch vertices and leaves, Graphs Combin. 35 (2019), no. 1, 231-238.

[13] M. M. Matthews and D. P. Sumner, Hamiltonian results in $K_{1,3}$-free graphs, J. Graph Theory 8 (1984), no. 1, 139-146.

[14] K. Ozeki and T. Yamashita, Spanning trees: A survey, Graphs Combin. 27 (2011), no. $1,1-26$.

[15] M. Tsugaki and Y. Zhang, Spanning trees whose stems have a few leaves, Ars Combin. 114 (2014), 245-256.

[16] S. Win, On a conjecture of Las Vergnas concerning certain spanning trees in graphs, Results Math. 2 (1979), no. 2, 215-224.

[17] Z. Yan, Spanning trees whose stems have a bounded number of branch vertices, Discuss. Math. Graph Theory 36 (2016), no. 3, 773-778. 
Pham Hoang Ha

Department of Mathematics, Hanoi National University of Education, 136 XuanThuy Street, Hanoi, Vietnam

E-mail address: ha.ph@hnue.edu.vn

Dang Dinh Hanh

Department of Mathematics, Hanoi Architectural University, Km10 NguyenTrai Str., Hanoi, Vietnam

E-mail address: hanhdd@hau.edu.vn

Nguyen Thanh Loan

Institute of Mathematics, Vietnam Academy of Science and Technology (VAST), Hanoi, Vietnam

E-mail address: ntloan@math.ac.vn 